\title{
Excimer Laser Processing of IGZO Thin Films for Transparent TFTs
}

\author{
Khairi. M. Abusabee ${ }^{\text {ab*, Khalid. M. Alajel }}{ }^{\text {a }}$, Salem. O. Elhamalic \\ ${ }^{a}$ Electrical and Computer Engineering Department, Faculty of Engineering, El-Mergib \\ University, Al-Khums, Libya \\ ${ }^{b}$ School of Science and Technology, Nottingham Trent University, Clifton Lane, Nottingham \\ NG11 8NS, UK \\ 'Electrical and Computer Engineering Department, Faculty of Engineering, Al-Asmarya Islamic \\ University, Zliteen, Libya
}

DOI: https://doi.org/10.21467/proceedings.2.28

* Corresponding author email: kmabusabee@elmergib.edu.ly

\begin{abstract}
The effects of post deposition annealing treatments on indium gallium zinc oxide IGZO thin films deposited by radio frequency RF magnetron sputtering at ambient temperature have been studied for application to thin film transistor TFT devices fabrication. Krypton Fluoride $\operatorname{KrF}(\lambda=248 \mathrm{~nm})$ excimer laser annealing ELA and low-temperature thermal annealing $\left(150^{\circ} \mathrm{C}\right)$ has been applied to IGZO films of $30 \mathrm{~nm}$ and $50 \mathrm{~nm}$ thickness as part of the fabrication process for TFT devices. The effect of annealing pre and post patterning of the IGZO channel layers was investigated. The results indicate that single pulse ultra rapid ELA is a viable technique for processing of the channel layers to provide TFT characteristics equivalent to or even better than that produced by a $150^{\circ} \mathrm{C}$ annealing for one hour. ELA treatment pre-patterning resulted in TFTs demonstrating a higher ON current and on/off current ratio relative to ELA treatment post-pattering. This could be attributed to surface defects introduced by the photolithographic patterning of the IGZO channel. In comparison, the thermally treated films exhibited better performance with the post-patterning thermal treatment.
\end{abstract}

Keywords: RF magnetron sputtering, excimer laser annealing, post pattering, pre pattering, thin film transistors, IGZO.

\section{Introduction}

Transparent amorphous oxide semiconductors TAOS are of a great importance in display technologies and are promising candidate materials for emerging applications in flexible transparent electronics. In particular, amorphous indium gallium zinc oxide based thin film transistors (IGZO TFTs) have attracted considerable attention [1,2], and have been demonstrated as switching devices in active matrix liquid crystal displays AMLCDs, and organic light emitting diode OLEDs based display panels[3, 4].

The first functional IGZO based TFTs reported in 2004 by Nomura et al, were fabricated on flexible substrates via pulsed laser deposition PLD. These devices were investigated as an

(C) 2018 Copyright held by the author(s). Published by AIJR Publisher in Proceedings of First Conference for Engineering

Sciences and Technology (CEST-2018), September 25-27, 2018, vol. 1.
This is an open access article under Creative Commons Attribution-NonCommercial 4.0 International (CC BY-NC 4.0) license, which permits any non-commercial use, distribution, adaptation, and reproduction in any medium, as long as the original work is properly cited. ISBN: $978-81-936820-5-0$ 
Excimer Laser Processing of IGZO Thin Films for Transparent TFTs

alternative to amorphous silicon (a-Si) and poly silicon (poly-Si) TFTs, because of the high field effect mobility that was demonstrated in the amorphous state $\left(>10 \mathrm{~cm}^{2} / \mathrm{Vs}\right)$ [1]. This mobility is attributed to the heavy metal cations with ( $n-1) \mathrm{d}^{10} \mathrm{~ns}^{0}(\mathrm{n} \geq 5)$ electronic configuration leading to a large overlap between adjacent cations orbitals [5]. Since the first reports on IGZO TFT research, there has been an increasing interest in studying the effect of post deposition annealing as a mechanism to improve the IGZO TFTs performance and stability. It has been reported that conventional post-deposition thermal annealing at $(\sim$ $400^{\circ} \mathrm{C}$ ) is an effective method to improve the IGZO TFTs performance [6], and stability [7], and reducing the tail state defects in IGZO film [5]. However, post thermal annealing at temperature higher than the maximum allowable temperature for heat resistance of plastic substrates $\left(\sim 150^{\circ} \mathrm{C}\right)$ is undesirable when considering low cost and flexible substrate applications. As an alternative to thermal annealing, photonic processing using lamps or laser irradiation is a method of interest for a range of thin films' applications where highly localised annealing and surface modification is imposed [8-12]. For example, Nakata et.al reported utilizing excimer laser annealing (ELA) to process $20 \mathrm{~nm}$ IGZO thin films for invertedstaggered bottom gate IGZO-TFTs deposited by RF magnetron sputtering, using two pulses from an XeCl excimer laser $(\lambda=308 \mathrm{~nm}) 25 \mathrm{~ns}$. IGZO-TFTs irradiated at laser fluences of 130 $\mathrm{mJ} / \mathrm{cm}^{2}$ exhibited ON current more than one order of magnitude higher than that of unannealed devices [8]. Ahn et.al fabricated bottom gate IGZO-TFT, using a $50 \mathrm{~nm}$ thick channel layer grown by RF magnetron sputtering at room temperature. The IGZO film was selectively laser annealed via a projection mask using an $\mathrm{XeCl}(\lambda=308 \mathrm{~nm})$ excimer laser in air ambient at ten pulses with a range of laser density from 0 to $250 \mathrm{~mJ} / \mathrm{cm}^{2}$. The electrical properties of the devices treated with laser energy density $130 \mathrm{~mJ} / \mathrm{cm}^{2}$ exhibited a channel mobility $21.79 \mathrm{~cm}^{2} / \mathrm{Vs}$, on/off ratio of $1.2 \times 10^{8}$, threshold voltage $\sim-0.15 \mathrm{~V}$. As the laser fluences increased to $130 \mathrm{~mJ} / \mathrm{cm}^{2}$ the resistivity of IGZO film decreased from $10^{4} \Omega \mathrm{cm}$ to $3.2 \times 10^{-3} \Omega \mathrm{cm}$ with carrier concentration $1.3 \times 10^{20} \mathrm{~cm}^{3}$, and hall mobility $15 \mathrm{~cm}^{2} / \mathrm{Vs}[11]$. It has also been reported by Zan et al [13] that $\mathrm{Nd}(\mathrm{YAG})$ laser (266 nm) or UV lamp irradiation $(172 \mathrm{~nm})$ supressed the instability of IGZO TFTs, and reduced the defects in IGZO film. Lim et.al demonstrated improvement of electrical properties of bottom gate $\mathrm{ZnO}$ TFT devices fabricated at low temperature $\left(200^{\circ} \mathrm{C}\right)[14]$.

For the investigation presented here, we have undertaken a comparison of low temperature $\left(150^{\circ} \mathrm{C}\right)$ thermal processing and the application of $\mathrm{KrF}$ photons with single pulse irradiation to study the effects on IGZO TFTs characteristics for devices utilising low temperature deposited IGZO thin films as the TFTs active layer. We are particularly interested in when the annealing treatment is applied in the fabrication process, pre or post IGZO channel pattering, and the effect this has on the resultant characteristics.

ISBN: 978-81-936820-5-0 


\section{Experimental procedure}

\subsection{Film deposition}

IGZO thin films of $30 \mathrm{~nm}$ and $50 \mathrm{~nm}$ thickness were deposited by RF magnetron sputtering from a ceramic target of $\mathrm{In}_{2} \mathrm{O}_{3}: \mathrm{Ga}_{2} \mathrm{O}_{3}: \mathrm{ZnO}$ molar ratio (1:1:1) and (purity 99.99\%) onto silicon dioxide coated silicon substrates. Deposition was performed at ambient temperature (with no intentional substrate heating) across a range of sputtering conditions by varying oxygen concentrations $\left(2 \% \mathrm{O}_{2} / \mathrm{Ar}, 5 \% \mathrm{O}_{2} / \mathrm{Ar}\right.$ ), and $\mathrm{RF}$ power (50 W, $100 \mathrm{~W}$ ) with deposition pressure $1.33 \mathrm{~Pa}$ to optimise the IGZO films for use in TFT device fabrication.

\subsection{Post- deposition annealing of IGZO thin films}

Annealing by thermal treatment at $150^{\circ} \mathrm{C}$ for one hour on a hotplate was compared with excimer laser processing undertaken at ambient temperature in air using a Lambda Physik $305 \mathrm{i}$ $248 \mathrm{~nm}, 20$ ns pulse KrF excimer laser, with a beam delivery system providing a homogenised $14 \mathrm{~mm} \times 14 \mathrm{~mm}$ uniform irradiation at the sample plane. Processing was undertaken at fluences in the range of $0 \mathrm{~mJ} / \mathrm{cm}^{2}$ to $175 \mathrm{~mJ} / \mathrm{cm}^{2}\left( \pm 6 \mathrm{~mJ} / \mathrm{cm}^{2}\right)$ using single pulse irradiation. The resultant IGZO thin films are characterised by four point probe (4PP) measurements, Hall Effect, and used in TFT test devices fabrication.

\subsection{IGZO-TFT fabrication}

Figure.1 shows the cross section of top gate bottom contacts IGZO-TFT device fabricated on $\mathrm{SiO}_{2} / \mathrm{Si}$ substrates as per the following process: (i) chromium (Cr) and gold ( $\mathrm{Au}$ ) deposited by evaporation for source and drain electrode contacts with thicknesses of $5 \mathrm{~nm}$ and $75 \mathrm{~nm}$ respectively and patterned by photolithography and lift off techniques; (ii) IGZO active layer deposited by RF magnetron sputtering with no intentional heating of the substrate, from an IGZO ceramic source target with $\operatorname{In}_{2} \mathrm{O}_{3}: \mathrm{Ga}_{2} \mathrm{O}_{3}: \mathrm{ZnO}$ molar ratio of (1:1:1) $99.9 \%$ purity at RF power of $50 \mathrm{~W}$, oxygen concentration $2 \% \mathrm{O}_{2} / \mathrm{Ar}$, at $1.33 \mathrm{~Pa}$ working pressure. The devices were divided into two sets. One was laser annealed before IGZO patterning, while the second set was laser annealed after IGZO patterning (in both cases, the samples were laser annealed as described in section 2.2. prior to the gate dielectric deposition). The IGZO layer was patterned via photolithography and wet etching producing IGZO channels with width (W) and length (L) of $1000 \mu \mathrm{m}$ and $5 \mu \mathrm{m}$ respectively. Alumina $\left(\mathrm{Al}_{2} \mathrm{O}_{3}\right)$ was deposited as the gate dielectric by atomic layer deposition ALD at $120^{\circ} \mathrm{C}$, and pattern by photolithography and lift off. Finally, a bilayer gate electrode of ( $\mathrm{Cr} / \mathrm{Au}, 5 / 75 \mathrm{~nm})$ was deposited by evaporation.

Proceedings of First Conference for Engineering Sciences and Technology (CEST-2018), vol. 1 
Excimer Laser Processing of IGZO Thin Films for Transparent TFTs

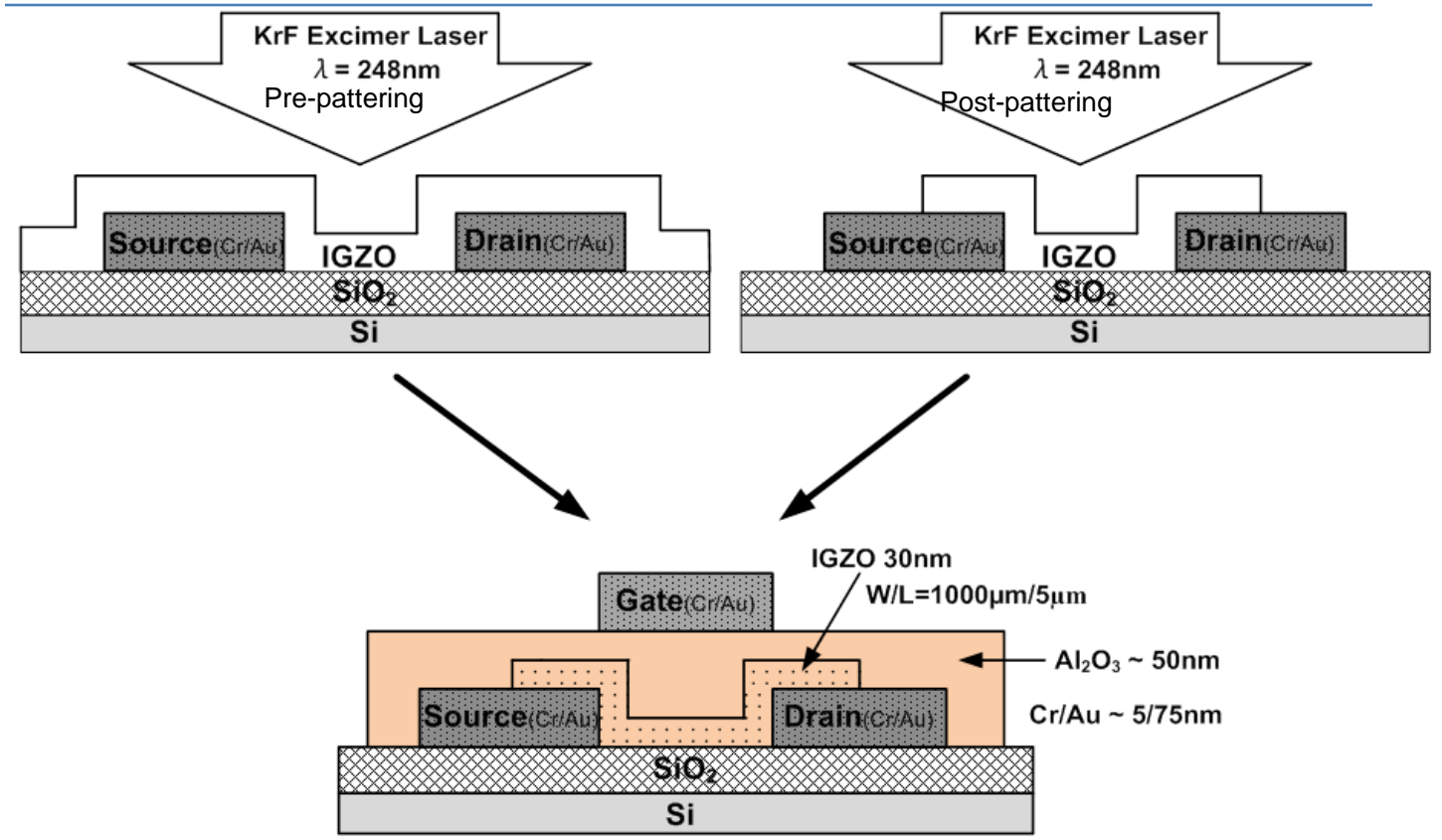

Figure 1: Schematic cross section of IGZO-TFT devices showing the concept of pre and post pattering laser anneal processing

\section{Results}

\subsection{Electrical properties of IGZO thin films}

Figure. 2 illustrates the sheet resistance of IGZO films deposited at an applied RF power of 50 $\mathrm{W}$ and various oxygen concentrations following laser annealing at various fluences using single pulse irradiation. The as-deposited IGZO films were highly resistive and not measurable using the four point probe system (i.e. they have sheet resistances $>5 \mathrm{MOhm} / \mathrm{sq}$ ) for all deposition conditions examined. A measurable sheet resistance was achieved at fluences of $\geq 75 \mathrm{~mJ} / \mathrm{cm}^{2}$, with the lowest sheet resistance of $1 \mathrm{kOhm} / \mathrm{sq}$ observed at a laser fluence of $100 \mathrm{~mJ} / \mathrm{cm}^{2}$ for all IGZO films studied. Processing at higher fluences led to an increase in sheet resistance coincident with visible damage of the film surface at these higher fluences. Similar trends were observed with films grown at RF power $100 \mathrm{~W}$. 


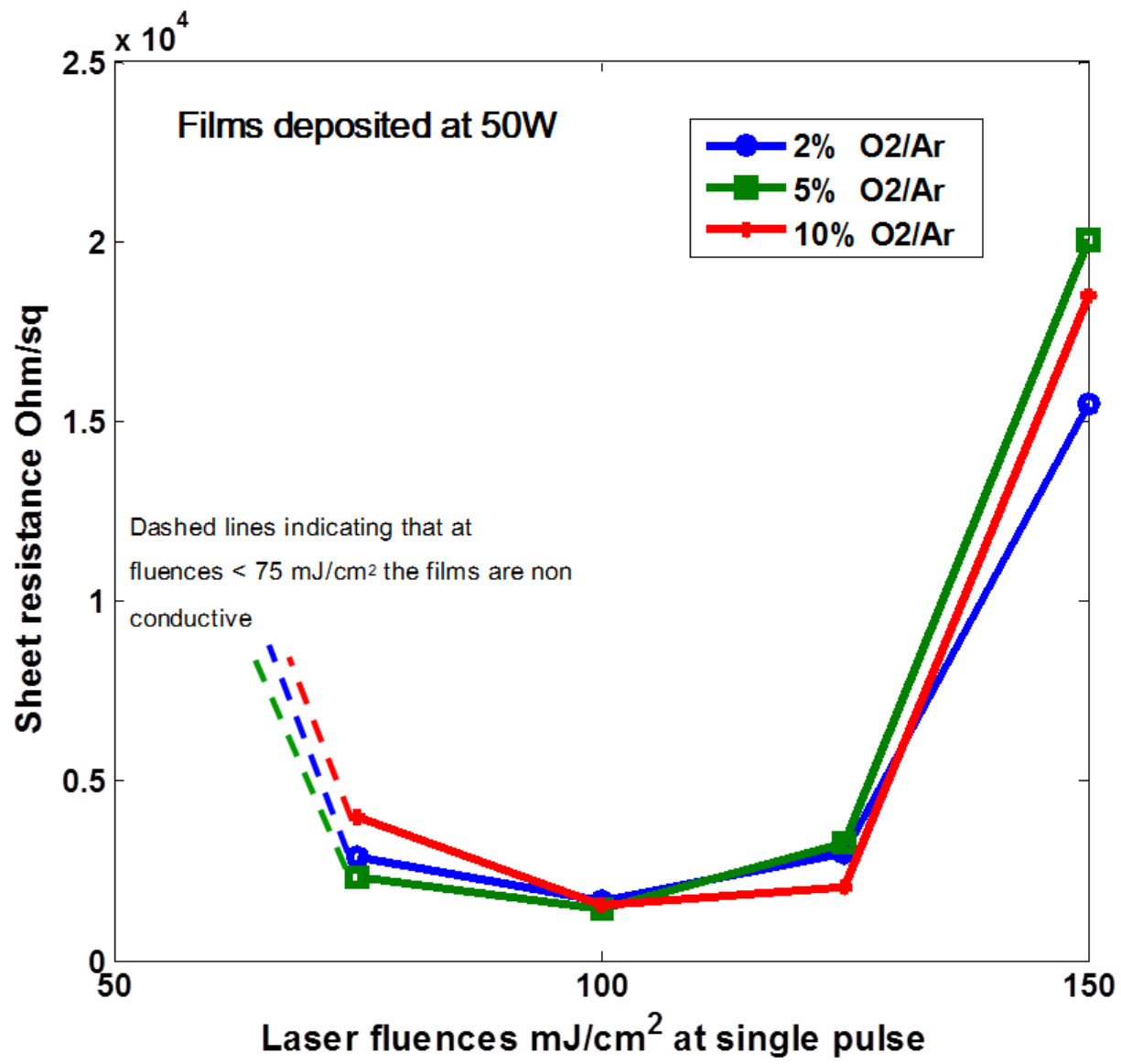

Figure 2: Sheet resistance of $30 \mathrm{~nm}$ thick IGZO films deposited at $50 \mathrm{~W}$ following laser processing irradiated at various fluences.

\subsection{Hall Effect Characterisation}

Figure. 3 shows the electrical characteristics as determined via room temperature Hall Effect measurement of the laser processed IGZO thin films, deposited at $50 \mathrm{~W}$ RF power, $2 \% \mathrm{O}_{2} / \mathrm{Ar}$, $50 \mathrm{~nm}$, as a function of laser fluence. The as-deposited and samples irradiated at laser fluences of $25 \mathrm{~mJ} / \mathrm{cm}^{2}$, and $50 \mathrm{~mJ} / \mathrm{cm}^{2}$ were too resistive to be measured, consistent with the four point probe measurements. At the higher fluences investigated, of $75 \mathrm{~mJ} / \mathrm{cm}^{2}$ and 100 $\mathrm{mJ} / \mathrm{cm}^{2}$, results were obtained indicating an increase in Hall mobility at higher fluences from $11.1 \mathrm{~cm}^{2} /$ Vs to $13.9 \mathrm{~cm}^{2} / \mathrm{Vs}$. At $175 \mathrm{~mJ} / \mathrm{cm}^{2}$, however, the mobility dropped to $1.67 \mathrm{~cm}^{2} / \mathrm{Vs}$, which again coincides with visible surface damage to the films. The drop in carriers mobility at higher fluences could be attributed to the onset of IGZO films crystallisation. The corresponding values of resistivity determined via Hall Effect measurement decreased from $5.92 \times 10^{-2}$ to $9.7 \times 10^{-3} \Omega . \mathrm{cm}$ with fluence increasing from 75 to $125 \mathrm{~mJ} / \mathrm{cm}^{2}$ due to a steady increase in carrier concentration that is reduced at higher fluences because of samples damage.

Proceedings of First Conference for Engineering Sciences and Technology (CEST-2018), vol. 1 
Excimer Laser Processing of IGZO Thin Films for Transparent TFTs

Regarding the thermally annealed samples, they were not measurable due to their high resistance to make contacts with the Hall effect equipment. However, the thermally annealed samples were suitable as the semiconducting channel layer in TFT fabrication, the results of which are described in section 3.3. Similarly, for TFT channel layer fabrication, the laser annealing fluences that produced functional TFT test devices were the lower fluence/higher resistance films processed at $\leq 75 \mathrm{~mJ} / \mathrm{cm}^{2}$.

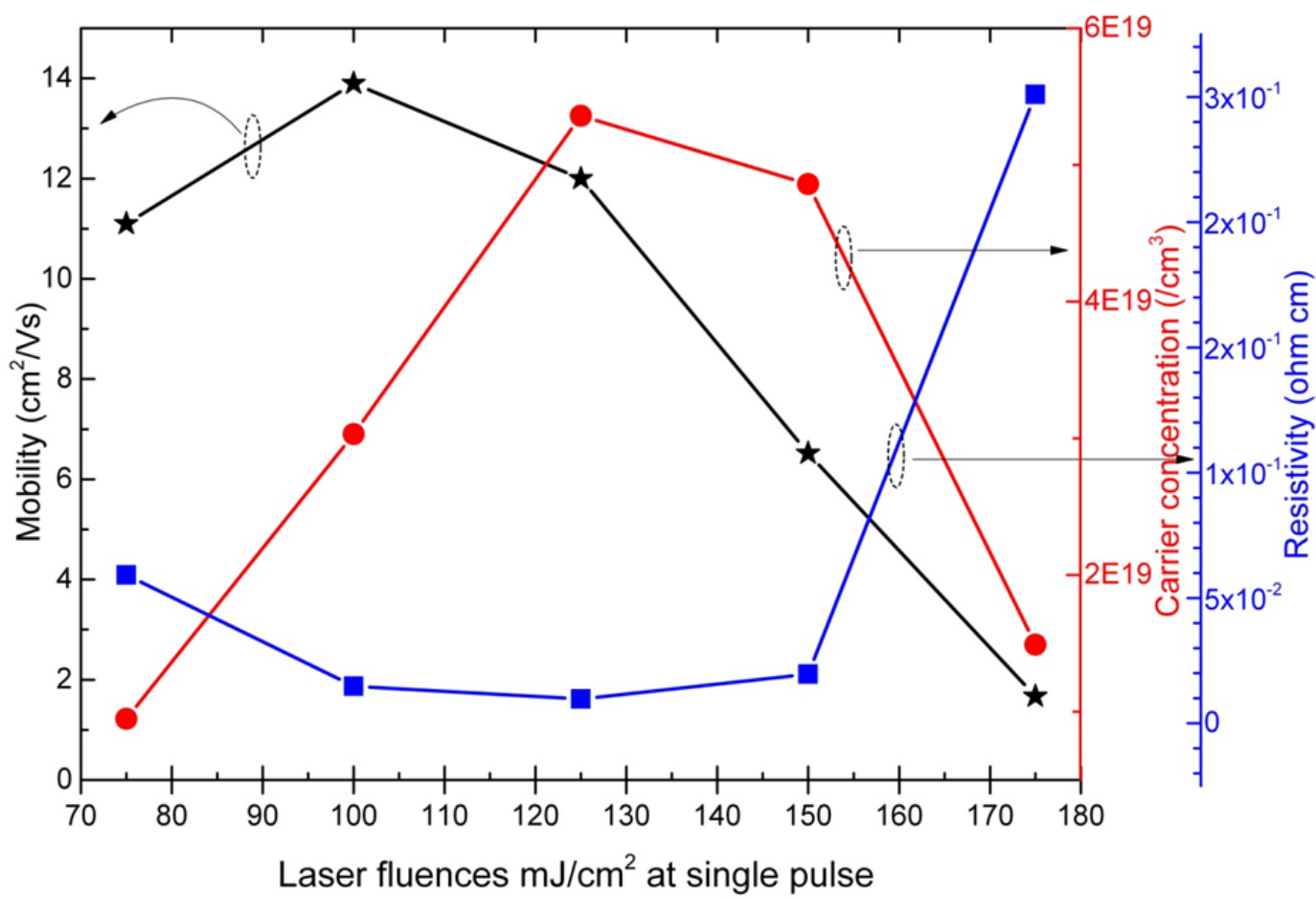

Figure3: Hall Effect characteristics, showing Hall mobility, carrier concentration, and resistivity of $50 \mathrm{~nm}$ thick IGZO thin films as a function of applied laser fluence.

For IGZO films with thickness of $30 \mathrm{~nm}$, no Hall mobility or conductivity could be detected for as-deposited films and laser irradiated films at various fluences. The high resistivity of laser annealed $30 \mathrm{~nm}$ film could be linked to the surface of the film being affected by atomspheric oxygen and changing the film properties and difficulties of achieving contacts with the Hall effect system. This is still a matter for investigation. However, it was reported by Nakata et.al in a comparison study, that the dependance of Hall mobility measurements and carrier density on laser fluence of laser annealed IGZO film by XeCl excimer laser $(\lambda=308 \mathrm{~nm})$ deposited at $20 \mathrm{~nm}$ where the highest Hall mobiliy achieved was $\sim 17 \mathrm{~cm}^{2} /$ Vs at laser energy density of about $180 \mathrm{~mJ} / \mathrm{cm}^{2}$ [16]. 


\subsection{Thermal annealed IGZO - TFTs result}

Figure 4 shows the transfer characteristics of IGZO-TFTs with the active channel is being IGZO films of $30 \mathrm{~nm}$ thick. The TFTs were thermally annealed at $150{ }^{\circ} \mathrm{C}$ in an air environment for 1 hour, before and after the channel layer patterning. The results are summarised in Table 1 as compared to the non-annealed device. There was a slight improvement in electrical performance of the thermally annealed IGZO TFTs pre IGZO patterning but with an increased OFF current. Whereas, thermally annealed IGZO TFTs post IGZO patterning show significant improvement of transfer characteristics. In both cases there is a negative shift in the threshold voltage, an improvement in the ON current, and a significant improvement in the On/Off current ratio for the post pattern annealed sample. In general, for the thermally annealed devices, the improvement in performance was better when annealed post IGZO patterning. Similar result trends were observed upon repetition of this exercise.

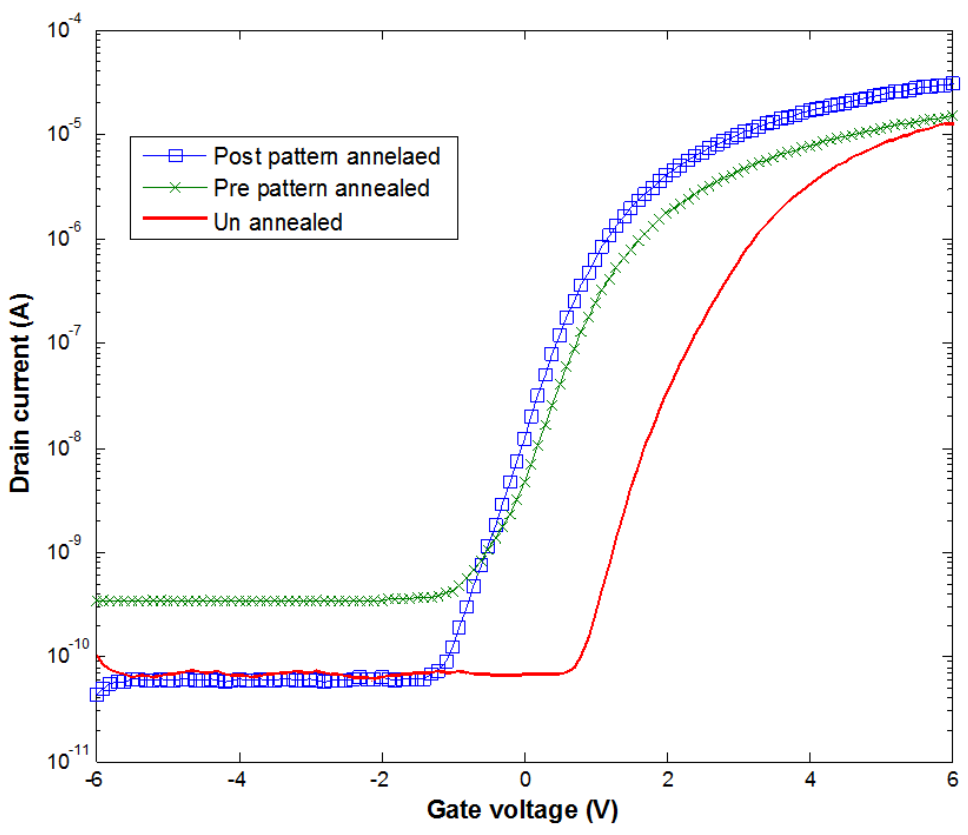

Figure 4: Transfer characteristics of IGZO-TFTs $(W / L=1000 \mu \mathrm{m} / 5 \mu \mathrm{m}), V_{D S}=1 \mathrm{~V}$ for samples that had been un annealed compared to samples thermal annealed at $150^{\circ} \mathrm{C}$ in air for 1 hour pre and post IGZO pattering.

Table 1: The effects of thermal annealing, at $150^{\circ} \mathrm{C}$ in air for 1 hour pre and post IGZO pattering, on IGZO -TFTs electrical properties.

\begin{tabular}{|l|c|c|c|c|c|}
\hline \multicolumn{1}{|c|}{ Sample } & $\mathrm{V}_{\text {th }}(\mathrm{V})$ & $\mu_{\mathrm{FE}}\left(\mathrm{cm}^{2} / \mathrm{Vs}\right)$ & $S(\mathrm{~V} / \mathrm{dec})$ & $\mathrm{I}_{\mathrm{D}} \mathrm{Max}(\mathrm{A})$ & $\mathrm{I}_{\mathrm{ON}} / \mathrm{I}_{\mathrm{OFF}}$ ratio \\
\hline Un-annaled & $3.52 \pm 0.14$ & $0.14 \pm 0.04$ & $0.41 \pm 0.14$ & $1.36 \times 10^{-5}$ & $1.48 \times 10^{5}$ \\
\hline $150^{\circ} \mathrm{C}$ pre patterning & $1.65 \pm 0.28$ & $0.10 \pm 0.03$ & $0.64 \pm 0.11$ & $1.54 \times 10^{-5}$ & $7.46 \times 10^{4}$ \\
\hline $150^{\circ} \mathrm{C}$ post patterning & $1.60 \pm 0.23$ & $0.16 \pm 0.09$ & $0.52 \pm 0.08$ & $2.75 \times 10^{-5}$ & $0.78 \times 10^{6}$ \\
\hline
\end{tabular}


Excimer Laser Processing of IGZO Thin Films for Transparent TFTs

Results indicated that thermal annealing at low temperature after IGZO channel layer patterning is more effective than before, which could be attributed to the effect on the interface between the IGZO layer and the dielectric layer by the photoresist heat treatment at $100^{\circ} \mathrm{C}$ for $1 \mathrm{~min}$ and $20 \mathrm{~s}$, since this would be a different effect when the photoresist is heated pre and post thermal anneal.

\subsection{Excimer laser annealed IGZO - TFTs result}

The TFT results for the laser processed samples are shown in Figure 5 which represents the characteristics from devices fabricated on the same substrate.

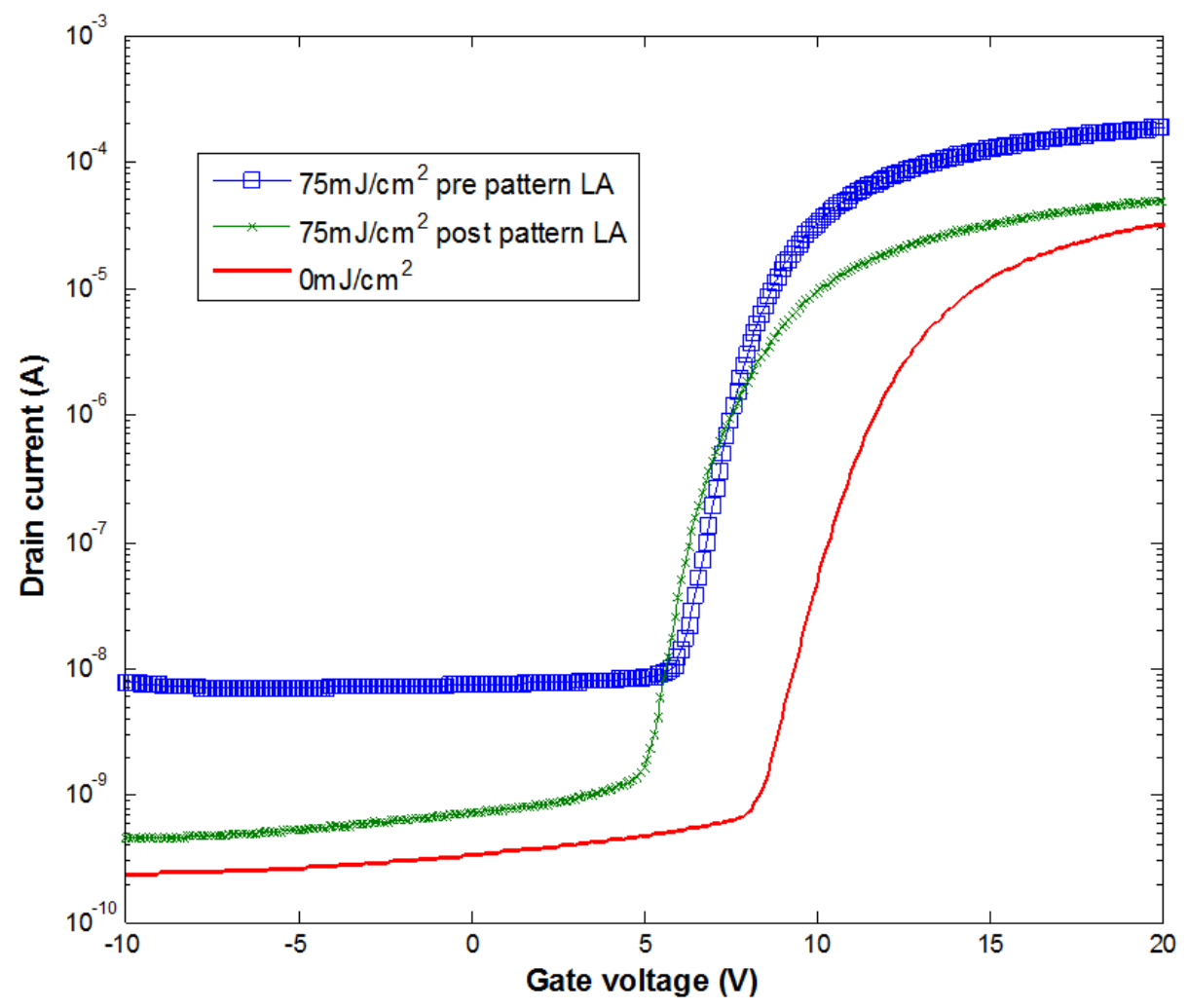

Figure 5: Transfer characteristics of IGZO-TFTs $(W / L=1000 \mu \mathrm{m} / 5 \mu \mathrm{m}), V_{D S}=1 \mathrm{~V}$, for samples un-annealed and laser annealed at a fluence of $75 \mathrm{~m} \mathrm{~J} / \mathrm{cm}^{2}$ in air.

As with the thermally annealed samples, the laser treated TFTs at laser energy density of 75 $\mathrm{mJ} / \mathrm{cm}^{2}$ with single pulse show a negative shift in threshold voltage and an increase of ON current. However, devices laser annealed pre IGZO pattern demonstrate higher ON and OFF current than the devices that were laser processed post to IGZO pattern. This could be assigned to surface defects introduced during photolithographic pattering deteriorating the performance of the laser annealed TFTs. The electrical properties of these devices are summarized in Table 2. 
Abusabee et al., CEST-2018, AIJR Proceedings 2, pp.233-243, 2018

Table 2: Properties of IGZO -TFTs laser annealed pre and post pattering of the active layer $30 \mathrm{~nm}$ thick.

\begin{tabular}{|l|c|c|c|c|c|}
\hline \multicolumn{1}{|c|}{ Sample } & $\mathrm{V}_{\text {th }}(\mathrm{V})$ & $\mu_{\mathrm{FE}}\left(\mathrm{cm}^{2} / \mathrm{Vs}\right)$ & $S(\mathrm{~V} / \mathrm{dec})$ & $\mathrm{I}_{\mathrm{D}} \operatorname{Max}(\mathrm{A})$ & IoN $_{\mathrm{O}}$ IoFF ratio \\
\hline Un- annealed & $12.1 \pm 0.7$ & $0.003 \pm 0.005$ & $0.70 \pm 0.04$ & $3.25 \times 10^{-5}$ & $1.58 \times 10^{5}$ \\
\hline $75 \mathrm{~mJ} / \mathrm{cm}^{2}$ pre pattern & $8.15 \pm 0.5$ & $0.64 \pm 0.69$ & $0.71 \pm 0.07$ & $2.00 \times 10^{-4}$ & $8.47 \times 10^{7}$ \\
\hline $75 \mathrm{~mJ} / \mathrm{cm}^{2}$ post pattern & $7.93 \pm 0.5$ & $0.25 \pm 0.21$ & $0.61 \pm 0.17$ & $5.01 \times 10^{-5}$ & $3.38 \times 10^{6}$ \\
\hline
\end{tabular}

Laser annealed IGZO-TFT devices with a channel layer $30 \mathrm{~nm}$ thick demonstrating very low field effect mobility. This is consistent with the observation that no Hall Effect measurements could be perfumed on $30 \mathrm{~nm}$ thick IGZO films at different laser energy densities, while laser processed $50 \mathrm{~nm}$ thick IGZO film exhibited Hall mobility as high as $13.9 \mathrm{~cm}^{2} / \mathrm{Vs}$.

\section{Discussion}

A significant drop in sheet resistance of IGZO thin films deposited without intentional substrate heating as a function of laser energy density for a single pulse is observed with a strong dependence on deposition parameters. Post deposition annealing of IGZO films leads to removal of weakly bonded oxygen atoms or oxygen combination contributing to a new equilibrium changing the electrical properties of IGZO films [13, 15]. The lowest sheet resistance post laser processing was observed at deposition conditions of low deposition pressure, low oxygen concentration, and high RF power. This can be attributed to the sputtered atoms' kinetic energy and thus their surface mobility on the substrate is being controlled with the deposition parameters. Hence, the resulting film prosperities are dependent on the films deposition parameters [2, 5]. Upon ELA, the resistivity of IGZO films was considerably reduced and the free electron density as well as the Hall Effect mobility were effectively enhanced as a function of laser fluence. These observations could be attributed to local atomic rearrangement, the amorphous structure randomness relaxation, and trap states density reduction. The local atomic rearrangement and structure relaxation caused by ELA heating, provided that no crystallisation occurs, would enhance the overlap among ns orbitals of the contained metals leading to better carrier mobility [2,11, 12]. The highest observed Hall effect mobility in this work was $13.9 \mathrm{~cm}^{2} /$ Vs obtained by ELA with single pulse at 100 $\mathrm{mJ} / \mathrm{cm}^{2}$, which is very promising to apply laser treated IGZO to IGZO TFTs.

For IGZO-TFT devices, thermal and laser annealing IGZO channel layer patterns result enhanced electrical performance of IGZO TFTs. This is originated from induced changes in IGZO layers upon annealing including reduction of scattering or trapping defects density on the bulk IGZO or at its interface, enhanced atomic bonding, and amorphous structure relaxation leading to enhanced electrical prosperities of IGZO [2, 11, 12]. laser irradiation is also expected to reduce the contact resistance between the IGZO channel layer and the source/drain electrodes due to reduction of IGZO channels resistivity and increasing the carrier concentration upon ELA. This would move the Fermi level in IGZO to higher positions towards the conduction band i.e. decreasing IGZO work function leading to better 
Excimer Laser Processing of IGZO Thin Films for Transparent TFTs

electrical contact between IGZO layer and S/D electrodes and thus better IGZO TFTs performance $[5,11]$. However, both annealing techniques resulted in negative shift in threshold voltage which could be attributed to the high defects density in the subgap density of states (DOS) pins the Fermi level, so no mobile carries. In addition, TFTs devices demonstrate high values of sub-threshold swing $S$ which is related to trap density in the subgap. High sub-threshold swing $S$ result in poor electrical properties such as, low speed and higher power consumption. Therefore, further investigations are needed to optimise the ELA application to IGZO TFTs fabrication using wide range of annealing parameters.

\section{Conclusions}

For the results presented here, the room temperature Hall Effect mobility of IGZO (50 nm thick) was effectively enhanced as the laser fluence was increased from $75 \mathrm{~mJ} / \mathrm{cm}^{2}$ to 100 $\mathrm{mJ} / \mathrm{cm}^{2}$ (single pulse) reaching values of $11.1 \mathrm{~cm}^{2} / \mathrm{Vs}$ and $13.9 \mathrm{~cm}^{2} / \mathrm{Vs}$ respectively.

Laser annealing of IGZO-TFT pre IGZO channel patterns result in relatively higher ON current than post pattern while thermally annealing post IGZO pattern demonstrate higher ON current. Hence, these results demonstrate that laser annealing is a powerful technique to modify the electrical properties of IGZO films grown at room temperature for TFTs applications.

\section{Acknowledgment}

We would like to acknowledge the UK Technology Stategy Board for support for this work as part of the SKTP project.

\section{References}

[1] W. S. Author, K. Nomura, H. Ohta, A. Takagi, T. Kamiya, M. Hirano and H. Hosono, "Room-temperature fabrication of transparent flexible thin-film transistors using amorphous oxide semiconductors," Nature, vol. 432, pp. 488-492, 2004.

[2] H. Yabuta, M. Sano, K. Abe, T. Aiba, T. Den, H. Kumomi, K. Nomura, T. Kamiya and H. Hosono, "High-mobility thin-film transistor with amorphous InGaZnO channel fabricated by room temperature RF-magnetron sputtering," Appl. Phys. Lett., vol. 89, pp. 112123, 2006.

[3] J. Y. Kwon, K. S. Son, J. S. Jung, T. S. Kim, M. K. Ryu, K. B. Park, B. W. Yoo, J. W. Kim, Y. G. Lee and K. C. Park, "Bottom-gate gallium indium zinc oxide thin-film transistor array for high-resolution AMOLED display," Electron Device Letters, IEEE, vol. 29, pp. 1309-1311, 2008.

[4] C. J. Kim, D. Kang, I. Song, J. C. Park, H. Lim, S. Kim, E. Lee, R. Chung, J. C. Lee and Y. Park, "Highly stable Ga2O3-In2O3-ZnO TFT for active-matrix organic light-emitting diode display application," in Electron Devices Meeting, 2006. IEDM'06. International, 2006, pp. 1-4.

[5] H. Hosono, K. Nomura, Y. Ogo, T. Uruga and T. Kamiya, "Factors controlling electron transport properties in transparent amorphous oxide semiconductors," J. Non Cryst. Solids, vol. 354, pp. 2796-2800, 5/1, 2008.

[6] K. Nomura, T. Kamiya, H. Ohta, M. Hirano, H. Hosono, "Defect passivation and homogenization of amorphous oxide thin-film transistor by wet O2 annealing , " Appl. Phys. Lett. 93 (2008) 192107.

[7] K. Nomura, T. Kamiya, Y. Kikuchi, M. Hirano, H. Hosono, "Comprehensive studies on the stabilities of a-In-GaZn-O based thin film transistor by constant current stress, " Thin Solid Films, 518 (2010) 3012.

[8] M. Nakata, K. Takechi, K. Azuma, E. Tokumitsu, H. Yamaguchi and S. Kaneko, "Improvement of InGaZnO4 thin film transistors characteristics utilizing excimer laser annealing," Applied Physics Express, vol. 2, pp. 1102, 2009. 
[9] N. Mitsuru, T. Kazushige, Y. Shinya, T. Eisuke, Y. Hirotaka and K. Setsuo, " Effects of Excimer Laser Annealing on InGaZnO4 Thin-Film Transistors Having Different Active-Layer Thicknesses Compared with Those on Polycrystalline Silicon, " Jpn J Appl Phys, 48 (2009) 115505.

[10] Y. Yang, S. S. Yang and K. Chou, "Characteristic Enhancement of Solution-Processed In-Ga-Zn Oxide ThinFilm Transistors by Laser Annealing," Electron Device Letters, IEEE, vol. 31, pp. 969-971, 2010.

[11] B. Du Ahn, W. H. Jeong, H. S. Shin, D. L. Kim, H. J. Kim, J. K. Jeong, S. H. Choi and M. K. Han, "Effect of excimer laser annealing on the performance of amorphous indium gallium zinc oxide thin-film transistors," Electrochemical and Solid-State Letters, vol. 12, pp. H430-H432, 2009

[12] C. Tsakonas, V. Kuznetsov, W. Cranton, N. Kalfagiannis, K. Abusabee, D. Koutsogeorgis, N. Abeywickrama and P. Edwards "Low temperature sputter-deposited ZnO films with enhanced Hall mobility using excimer laser post-processing," J. Phys. D: Appl. Phys. vol. 50, pp. 485306 (15). 2017

[13] H. W. Zan, W. T. Chen, C. W. Chou, C. C. Tsai, C. N. Huang and H. W. Hsueh, "Low temperature annealing with solid-state laser or UV lamp irradiation on amorphous IGZO thin-film transistors, "Electrochemical and Solid-State Letters, vol. 13, pp. H144-H146, 2010.

[14] S. Lim, J. M. Kim, D. Kim, C. Lee, J. S. Park and H. Kim, "The Effects of UV Exposure on Plasma-Enhanced Atomic Layer Deposition ZnO Thin Film Transistor, "Electrochemical and Solid-State Letters, vol. 13, pp. H151H154, 2010.

[15] A. Suresh, P. Gollakota, P. Wellenius, A. Dhawan and J. F. Muth, "Transparent, high mobility InGaZnO thin films deposited by PLD," Thin Solid Films, vol. 516, pp. 1326-1329, 2008

[16] M. Nakata, K. Takechi, S. Yamaguchi, E. Tokumitsu, H. Yamaguchi and S. Kaneko, "Effects of excimer laser annealing on InGaZnO4 thin-film transistors having different active-layer thicknesses compared with those on polycrystalline silicon," Jpn J Appl Phys, vol. 48, pp. 115505-115505-6, 2009. 